\title{
Variantes Anatómicas en el Canal Mandibular en Adultos Jóvenes Mayores de 30 Años
}

\author{
Anatomical Variants in the Mandibular Canal in Young Adults Over 30 Years
}

\author{
Álvaro Sirera Martín \& Andrés Martínez Almagro Andreo
}

SIRERA, M.A. \& MARTÍNEZ-ALMAGRO ANDREO, A. Variantes anatómicas en el Canal mandibular en adultos jóvenes mayores de 30 años. Int. J. Morphol., 38(4):899-902, 2020.

RESUMEN: La correcta identificación del canal mandibular es esencial cuando se pretende realizar procedimientos quirúrgicos del sector posterior o incluso odontología clínica. La tomografía computarizada de haz cónico (CBCT) utiliza diferentes planos para la evaluación de este. Para conocer las variaciones anatómicas del canal mandibular se lleva a cabo un estudio en 100 adultos mayores de 30 años, donde la ubicación, la forma y la orientación se estudia mediante la tomografía computarizada de haz cónico. Así, el principal objetivo de nuestro estudio es conocer la variabilidad y tipo de presentación del canal bífido en el Canal mandibular en pacientes dentados o parcialmente edéntulos adultos mayores de 30 años.

PALABRAS CLAVE: Anatomía; Mandíbula; Tomografía; Adulto.

\section{INTRODUCCIÓN}

El canal mandibular se presenta como una estructura ósea bilateral que plantea diferentes retos de relevancia clínica. Uno de ellos es la posible bifurcación del canal dentario inferior (Wadu et al., 1997). Esta variante anatómica que se observa como una división del nervio en dos ramas, cada una en su propio canal, puede presentarse de diferentes formas, uni o bilateralmente. Naitoh et al. (2009) describieron la presencia de canales mandibulares bífidos en el 2009 lo hicieron mediante $\mathrm{CBCT}$, donde consideraron cuatro tipos (Fig. 1).

\section{MATERIAL Y MÉTODO}

Para llevar a cabo nuestra investigación nos hemos planteado realizar un estudio descriptivo observacional retrospectivo transversal, siendo la población de estudio los pacientes que asistieron a un centro clínico de imagen biomédica situado en la provincia de Alicante, Affidea Clínica Tesla (2018). Seleccionamos una muestra basada en las tomografías computarizadas rescatadas de las historias clínicas de los pacientes que realizaron una consulta odontológica, desde el año 2005, y que presentaron las si-

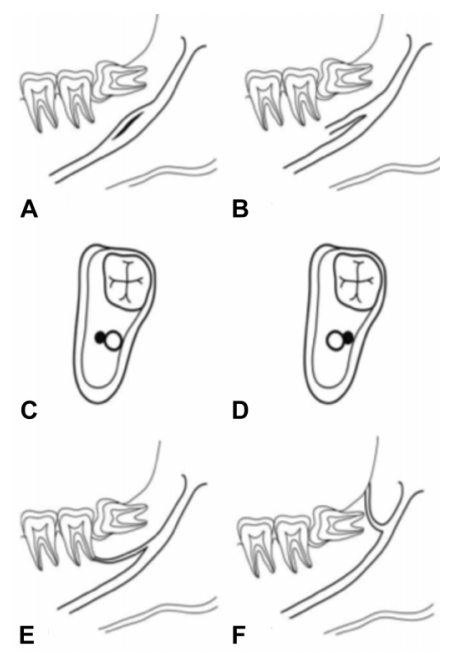

Fig. 1. Clasificación de la bifurcación del canal mandibular según Naitoh y colaboradores (Naitoh $\mathrm{M}$ et al (2009)), en 4 tipos: canal anterior, con o sin confluencia (A y B); bucolingual, vinculado a la pared bucal o lingual de la mandíbula (C y D); dental, alcanzando la raíz dental (E); y retromolar, en relación al tercer molar $(\mathrm{F})$. Imagen tomada Kang y cols (Kang J et al (2014))

guientes características o criterios de selección: Historias clínicas de los pacientes asistentes al centro clínico Tesla en Elche; participación voluntaria y consentimiento informado; pacientes sanos y pacientes dentados o parcialmente edéntulos, no gestantes, mayores de 30 años de edad, con ausencia de alteración patológica en la mandíbula y/o canal mandibular; ausencia de intervención quirúrgica y/o altera- 
ciones patológicas en el maxilar inferior y/o de asimetrías importantes en la región craneal.

Realizamos la selección de las historias clínicas, junto con el técnico del centro biomédico, quien nos dispuso la muestra de estudio, aplicando los criterios de selección y pudiendo participar en el estudio sólo pacientes que firmaron el consentimiento informado para con la clínica. Con el objetivo de mantener el anonimato y privacidad de la historia clínica, los únicos datos cedidos por la clínica fueron edad y sexo del paciente. El tratamiento, comunicación y cesión de los datos se realizó conforme a lo dispuesto por la Ley Orgánica 15/1999, de 13 de diciembre, de Protección de Datos de Carácter Personal (BOE núm. 298 de 14 de diciembre de 1999) (Boletín oficial del Estado, 1999), pudiendo el participante acceder a sus datos, corregirlos o cancelarlos.

La obtención de la imagen fue realizada a través de un registro radiográfico mediante tomografía computarizada de haz cónico en el centro clínico de referencia del estudio mediante un equipo Planmeca PROMAX 3D Classic con cefalostato, estando el paciente en bipedestación durante la toma de la imagen.

El voltaje del tubo de rayos $\mathrm{X}$ fue de $90 \mathrm{kVp}$, la corriente de $12 \mathrm{mAs}$ y el tiempo de exposición de $15 \mathrm{~s}$. Para gestionar las imágenes obtenidas utilizamos el programa de gestión de imagen Planmeca Romexis Viewer. A partir de estas presentaciones identificamos una serie de puntos anatómicos de referencia y determinamos las distancias en dos planos con respecto a éste y así ubicar con mayor precisión su posición, morfología y comportamiento con las estructuras blandas que discurren por este Canal, determinando las siguientes líneas:

- Línea 1: línea vertical en el plano sagital, del margen superior del foramen al margen inferior de la escotadura mandibular.

- Línea 2: línea vertical en el plano sagital, del margen inferior del foramen al margen inferior del tramo horizontal del cuerpo de la mandíbula.

- Línea 3: línea horizontal en el plano sagital, del margen anterior del foramen al margen anterior de la rama mandibular.

- Línea 4: línea horizontal en el plano sagital, del margen posterior del foramen al margen posterior de la rama mandibular.

- Línea 5: línea vertical en el plano sagital, del margen superior del foramen al margen inferior del espacio alveolar dentario.

- Línea 6: línea vertical en el plano sagital, del margen inferior del foramen al margen inferior de la rama mandibular.
Las variables de estudio usadas han sido el sexo, la edad, el tipo de dentición, tipo de canal mandibular y las líneas de referencia descritas. Para el análisis estadístico, hemos realizado un análisis descriptivo de todas las variables mediante cálculo de frecuencias para las cualitativas, y valor mínimo, máximo, medio y desviación estándar para las cuantitativas.

Hemos analizado los factores asociados al sexo y a la presencia de canal bífido mediante tablas de contingencia, aplicando el test exacto de Fisher para las variables cualitativas, y comparación de valores medios para las cuantitativas, aplicando el test no paramétrico U de Mann-Whitney para las variables de 2 categorías, y el test de Kuskall-Wallis para las variables de más de 2 categorías.

Finalmente, hemos analizado la asociación de la edad con las variables cuantitativas mediante la estimación del coeficiente no paramétrico Rho de Spearman. Y, en relación a la comparativa entre lados, utilizamos el test $\mathrm{T}$ de Student para muestras relacionadas, y el coeficiente de concordancia de Kappa.

Los análisis han sido realizados mediante el programa SPSS v.25.

\section{RESULTADOS}

Tras nuestro proceso de selección, analizamos un total de 100 sujetos - 200 hemimandíbulas-, con una edad media de 44,5 años -rango de 31 a 55 años-. En relación al sexo, el 52 $\%$ de los participantes fueron mujeres; y en relación al tipo de dentición, un $61 \%$ de los casos presentó una dentición completa. En relación de pacientes con presencia de una bifurcación del canal mandibular, en ambos casos encontramos una distribución similar, mostrando un porcentaje del 14 y $15 \%$ de los pacientes una bifurcación del canal mandibular -y con un mayor número de casos de aparición a nivel retromolar (9 $\%$ en el lado derecho y $8 \%$ en el izquierdo), valores similares a los descritos por Rashsuren et al. (2014) (Fig. 2).

Las Figuras 3 y 4 muestran gráficamente la distribución de los resultados en función del sexo y de la edad.

La Figura 3 refleja los valores en \%, sobre una muestra de 52 mujeres -con 44 y 43 ausencias de bifurcación, 5 y 6 retromolares, 2 y 1 anterior, 1 y 2 dental en los lados izquierdo y derecho respectivamente- y 48 hombres -con 41 y 43 ausencias de bifurcación, 3 y 3 retromolares, 4 y 2 anteriores y ninguna dental, en los lados izquierdo y derecho respectivamente. 
En ellos observamos que no existieron diferencias significativas entre sexos (p-valor $<0,489$ lado derecho y $<0,601$ lado izquierdo).

Estos datos muestran -sin existir diferencias significativas entre sí- una tendencia a asociar una bifurcación anterior en personas de mayor edad, con una surgencia retromolar en las edades menores, dentro de nuestro rango de edad de la muestra, pacientes entre 30 y 55 años.

Tipo de canal mandibular

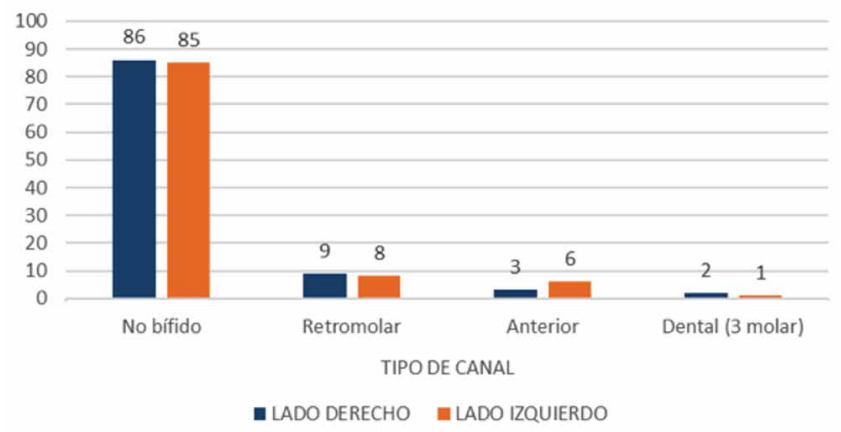

Fig. 2. Presencia de canal bífido mandibular (en \%).

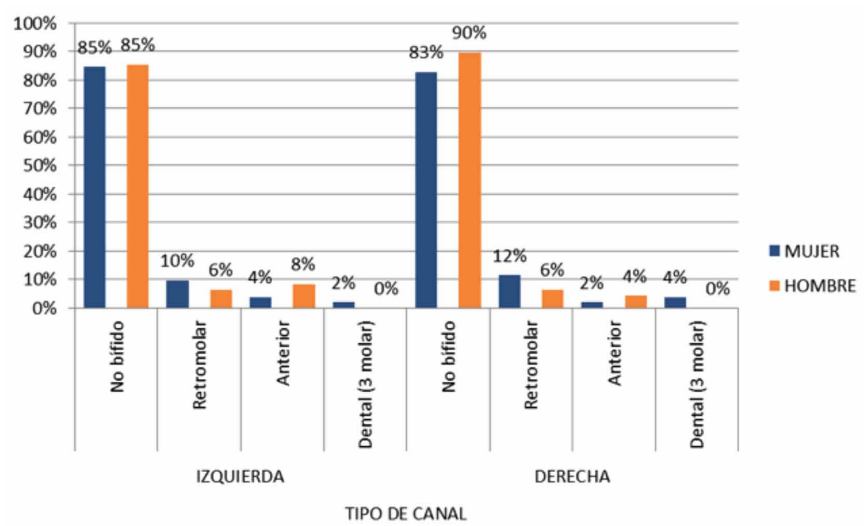

Fig. 3. Presencia de canal bífido mandibular en función del sexo (en \%).

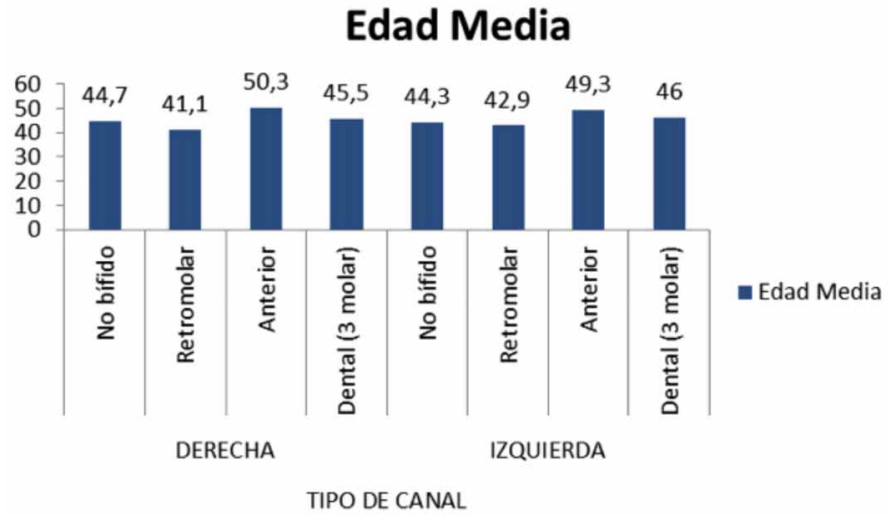

Fig. 4. Presencia de canal bífido mandibular en función de la edad (en años).

\section{DISCUSIÓN}

La incidencia de canales mandibulares bífidos se encuentra en el rango de 0.08-0.95\% en estudios que utilizan radiografías panorámicas (Nortje et al., 1977; Langlais et al., 1985; Grover \& Lorton, 1983; Zografos et al., 1990). Por otro lado, en estudios que usan CBCT, mostraron unos valores de existencia de esta bifurcación en el rango de 15.6-64.8 \% (de Oliveira-Santos et al., 2012; Kuribayashi et al., 2010; Naitoh et al., 2010; Orhan et al., 2011).

La comparación de ambas técnicas de imagen apoya la validez del uso de la CBCT como principal opción a la hora de establecer un estudio de la anatomía de la región cráneo-facial, especialmente con la aplicación en el ámbito odontológico.

Estos datos nos plantean la relevancia de conocer la disposición del canal mandibular y cómo éste puede afectar en la práctica clínica. Conocer la trayectoria del canal dentario inferior es importante debido a que cualquier bifurcación puede ser la causante de una errónea praxis anestésica. Un trabajo realizado por Vinckier (2000), estimó un $10 \%$ de fracaso del bloqueo del nervio dentario inferior, siendo de suma importancia ya que, en la práctica diaria de la odontología, la anestesia local realizada por el odontólogo tiene que ser eficaz, técnicamente correcta y realizada con un anestésico de garantía.

Detectar la disposición de las distintas estructuras anatómicas es necesario en la práctica clínica odontológica diaria, siendo un elemento relevante de nuestro trabajo conocer la de variación del Canal dentario inferior ya que, como ejemplo, la patología del tercer molar adquiere muchas veces un papel protagonista y un reto importante. Aun coincidiendo con los datos de otros estudios en la relación nervio dentario inferior/tercer molar, el potencial riesgo de lesiones del nervio dentario inferior o del paquete vascular requiere un conocimiento preciso de las estructuras anatómicas adyacentes en general y de ciertos detalles anatómicos en particular (Blaeaser et al., 2003; Bell, 2004).

El canal bífido de la parte izquierda se asocia significativamente con el nivel de surgencia vertical tanto de la parte derecha como de la izquierda, de forma que los que no presentan canal bífido se asocian en mayor medida con surgencia inferior, y los que presentan canal bífido con los de surgencia a nivel. La surgencia superior se presenta por igual en bífidos y no bífidos. El canal bífido de la parte derecha no se asocia significativamente con el resto de variables. 


\section{CONCLUSIONES}

Existe una variabilidad baja, aunque determinante en la presentación del Canal mandibular, con un porcentaje del $14 \%$ de casos en el lado derecho y del $15 \%$ de casos en el lado izquierdo que presentaron un canal bífido. En la presentación de la bifurcación encontrada del canal mandibular de nuestra muestra la dirección retromolar fue la más frecuente -17 casos sobre 200 hemimandíbulas-, seguida de una dirección anterior -9 casos de 200- y finalmente la dirección dental, a nivel del tercer molar -3 casos de 200-. Es necesario continuar con este campo de investigación para lograr una mayor eficiencia clínica y científica.

SIRERA, M. A. \& MARTÍNEZ-ALMAGRO ANDREO, A. Anatomical variants in the mandibular canal in young adults over 30 years. Int. J. Morphol., 38(4):899-902, 2020.

SUMMARY: The correct identification of the mandibular canal is essential when trying to perform surgical procedures in the posterior sector or even clinical dentistry. Computed tomography (CBCT) uses different planes for the evaluation of this. In order to know the anatomical variations of the mandibular canal, a study is carried out in 100 adults over 30 years of age, where the location, shape and orientation is studied by cone beam computed tomography. The main objective of our study is to know the variability and type of presentation of the bifid canal in the mandibular duct in dentate or partially edentulous adult patients over 30 years.

KEY WORDS: Anatomy; Mandible; Tomography; Adult.

\section{REFERENCIAS BIBLIOGRÁFICAS}

Affidea Clínica Tesla. Elche, Affidea, 2018. Disponible en: https://affidea.es/ portfolio-items/affidea-clinica-tesla/

Bell, G. W. Use of dental panoramic tomographs to predict the relation between mandibular third molar teeth and the inferior alveolar nerve. Radiological and surgical findings, and clinical outcome. Br. J. Oral Maxillofac. Surg., 42(1):21-7, 2004.

Blaeser, B. F.; August, M. A.; Donoff, R. B.; Kaban, L. B. \& Dodson, T. B. Panoramic radiographic risk factors for inferior alveolar nerve injury after third molar extraction. J. Oral Maxillofac. Surg., 61(4):417-21, 2003.

Boletín Oficial del Estado. Ley Orgánica 15/1999, de 13 de diciembre, de Protección de Datos de Carácter Personal. Internet. Madrid, Jefatura del Estado, 1999. Disponible en: http://www.boe.es/boe/dias/1999/12/ 14/pdf./A43088-43099.pdf

de Oliveira-Santos, C.; Souza, P. H.; de Azambuja Berti-Couto, S.; Stinkens, L.; Moyaert, K.; Rubira-Bullen, I. R. \& Jacobs, R. Assessment of variations of the mandibular canal through cone beam computed tomography. Clin. Oral. Investig., 16(2):387-93, 2012.

Grover, P. S. \& Lorton, L. Bifid mandibular nerve as a possible cause of inadequate anesthesia in the mandible. J. Oral Maxillofac. Surg., 41(3):177-9, 1983.
Kuribayashi, A.; Watanabe, H.; Imaizumi, A.; Tantanapornkul, W.; Katakami, K. \& Kurabayashi, T. Bifid mandibular canals: cone beam computed tomography evaluation. Dentomaxillofac. Radiol., 39(4):2359, 2010 .

Langlais, R.; Broadus, R. \& Glass, B. J. Bifid mandibular canals in panoramic radiographs. J. Am. Dent. Assoc., 110(6):923-6, 1985.

Naitoh, M.; Hiraiwa, Y.; Aimiya, H. \& Ariji, E. Observation of bifid mandibular canal using cone-beam computerized tomography. Int. J. Oral Maxillofac. Implants, 24(1):155-9, 2009.

Naitoh, M.; Nakahara, K.; Suenaga, Y.; Gotoh, K.; Kondo, S. \& Ariji, E. Comparison between cone-beam and multislice computed tomography depicting mandibular neurovascular canal structures. Oral Surg. Oral Med. Oral Pathol. Oral Radiol. Endod., 109(1):e25-31, 2010.

Nortje, C. J.; Farman, A. G. \& Grotepass, F. W. Variations in the normal anatomy of the inferior dental (mandibular) canal: a retrospective study of panoramic radiographs from 3612 routine dental patients. Br. J. Oral Surg., 15(1):55-63, 1977

Orhan, K.; Aksoy, S.; Bilecenoglu, B.; Sakul, B. U. \& Paksoy, C. S. Evaluation of bifid mandibular canals with cone-beam computed tomography in a Turkish adult population: a retrospective study. Surg. Radiol. Anat., 33(6):501-7, 2011.

Rashsuren, O.; Choi, J. W.; Han, W. J. \& Kim, E. K. Assessment of bifid and trifid mandibular canals using cone-beam computed tomography. Imaging Sci. Dent., 44(3):229-36, 2014.

Vinckier, F. What is the cause of failure of local anesthesia? Rev. Belge. Med. Dent. (1984), 55(1):41-50, 2000.

Wadu, S. G.; Penhall, B. \& Townsend, G. C. Morphological variability of the human inferior alveolar nerve. Clin. Anat., 10(2):82-7, 1997.

Zografos, J.; Kolokoudias, M. \& Papadakis, E. The types of the mandibular canal. Hell. Period. Stomat. Gnathopathoprosopike. Cheir., 5(1):1720, 1990.

Dirección para correspondencia:

Álvaro Sirera Martín

Cátedra de Anatomía Humana

Facultad de Ciencias de la Salud

Universidad Católica de Murcia

Avda. de los Jerónimos, 135. 30107

Guadalupe, Murcia

ESPAÑA

Email: asirera@alu.ucam.edu

Recibido : 05-02-2020

Aceptado: $16-03-2020$ 